Indexed by

\title{
Scopus
}

\section{THE USE OF RADAR TECHNOLOGIES IN THE HYDRAULIC ENGINEERING IN SEISMIC ZONES}

Crossref

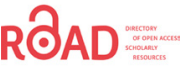

KOBSON

I8 Google

\section{Zhanar O. Oralbekova}

L.N. Gumilyov Eurasian National University, Department of Computer and Software

Engineering, Nur-Sultan, Republic of Kazakhstan

\section{Aigul D. Adamova}

S. Seifullin Kazakh Agrotechnical University, Department of Computing Engineering and Software, Nur-Sultan, Republic of Kazakhstan

\author{
Gulnur A. Tyulepberdinova \\ Al-Farabi Kazakh National \\ University, Department of \\ Informatics, Almaty, \\ Republic of Kazakhstan
}

\author{
Gulnur G. Gaziz \\ Al-Farabi Kazakh National \\ University, Department of \\ Informatics, Almaty, \\ Republic of Kazakhstan
}

\author{
Bakytgerey B. Sholpanbaev \\ Abai Kazakh National \\ Pedagogical University, \\ Institute of Mathematics, \\ Physics and Informatics, \\ Almaty, \\ Republic of Kazakhstan
}

Key words: groundwater, destructibility of hydraulic facilities, water storage, air traffic, radar systems

doi:10.5937/jaes0-30937

Cite article:

Oralbekova O. Z., Tyulepberdinova A. G., Gaziz G. G., Adamova D. A., Sholpanbaev B. B., (2021) THE USE OF RADAR TECHNOLOGIES IN THE HYDRAULIC ENGINEERING IN SEISMIC ZONES, Journal of Applied Engineering Science, 19(4), 1040 - 1048, DOI:10.5937/ jaes0-30937

Online aceess of full paper is available at: www.engineeringscience.rs/browse-issues 


\title{
THE USE OF RADAR TECHNOLOGIES IN THE HYDRAULIC ENGINEERING IN SEISMIC ZONES
}

\author{
Zhanar O. Oralbekova ${ }^{1 *}$, Gulnur A. Tyulepberdinova², Gulnur G. Gaziz², Aigul D. Adamova ${ }^{3}$, Bakytgerey B. \\ Sholpanbaev ${ }^{4}$ \\ ${ }^{1}$ L.N. Gumilyov Eurasian National University, Department of Computer and Software Engineering, \\ Nur-Sultan, Republic of Kazakhstan \\ ${ }^{2}$ Al-Farabi Kazakh National University, Department of Informatics, Almaty, Republic of Kazakhstan \\ ${ }^{3}$ S. Seifullin Kazakh Agrotechnical University, Department of Computing Engineering and Software, \\ Nur-Sultan, Republic of Kazakhstan \\ ${ }^{4}$ Abai Kazakh National Pedagogical University, Institute of Mathematics, Physics and Informatics, Almaty, \\ Republic of Kazakhstan
}

Hydraulic structures are designed in a standard way if it is necessary to regulate the water course and maintain the volume of irrigation. The obstacles to the construction of such structures are mainly technical and economic, when instead of building a new hydraulic complex, it is gradually modernised. Consideration of the issues of creating complexes of hydraulic structures leads to the statement of a fact that the water balance of the territory changes. The novelty of the study is determined by the fact that hydraulic structures can be used as prerequisites for the development of qualitatively new programmes for runoff regulation. The authors note that this is particularly important for seismologically unstable areas. It is necessary to lay out plans taking into account not only the possible seismological load, but also the modes and technologies that are used to modernise the already operating hydraulic structures. The practical significance of the study is determined by the fact that the development of hydrotechnical complexes makes it possible to create a system not only for improving the quality of the water balance, but also performs technological support for the safety of the technologies used.

Key words: groundwater, destructibility of hydraulic facilities, water storage, air traffic, radar systems

\section{INTRODUCTION}

At the end of the 20th century and the beginning of the $21 \mathrm{st}$, there was a significant increase in the number of natural and man-made disasters, causing economic damage in hundreds of billions of dollars, and significantly affecting the natural environment of the Earth and humanity as a whole [1]. Among natural and man-made disasters, with their enormous harm and impact on large areas, there are spills of oil products on the sea surface during extraction and transportation, the flooding of territories, that is, a rise in the level of groundwater and waterlogging of soils caused by economic and industrial activities, subsurface peat fires, etc. [2].

Therefore, for example, the problem of flooding in terms of its scale and dynamics of development has recently acquired signs of a potential threat to human habitation area [3]. At the same time, a water table rise is observed almost everywhere, due to an increase in water level in rivers during the construction of impounding reservoirs, channel dams, shipping canals and other hydraulic structures. It is also influenced by saturation of soils during water seepage through the bottom and banks of canals, leaks from water supply and sewerage networks [4]. According to various estimates, flooding affects up to $30 \%$ of the population, $60 \%$ of industrial-urban agglomerations, almost all developed mining areas and industrial sites of thermal power plants [5].
Currently, numerous radar systems are successfully operating in space: RADARSAT -1, -2 (Canada), ENVISAT ASAR, ERS-1, -2 (ECA), ALOS PALSAR (Japan), Lacross $2, \ldots-5$ (USA), TerraSARX1, -X2, Sar-Lupe $-1 \ldots$ -5 (Germany), COSMO SkyMed (Italy), TECSAR (Israel), JianBing -5 (China) and many others, which opened up wide opportunities for remote sensing soil moisture in various regions of the Earth [6]. However, radar systems have significant limitations [7]. Thus, surface roughness has a significant effect on radar scatter; this makes it difficult to assess the soil moisture using active radar [8]. The radar signal is also quenched and scattered by vegetation; which limits the use of active radar techniques only on surfaces with little to no vegetation [9]. The penetration distance of radio waves varies depending on the soil dielectric constant, the angle of incidence and the frequency of exposure, however, the complex senses moisture in the soil layer several centimetres thick, which does not allow using them to observe the flooding of deeper soil layers [10].

For remote sensing of temperature anomalies caused by waterlogging of depth layers in soil and subsurface waters, space-borne and airborne IR devices are widely used [11]. The problems encountered when assessing moisture conditions are caused by a variety of sources of electromagnetic radiation, captured by the IR sensor 
(for example, soil, foliage of vegetation and wood material, debris, etc.), the range of influence of variables that can determine the flux of IR radiation (for example, surface moisture, incident radiation, wind, albedo, thermal inertia, etc.), and the transmission of emitted radiation through the atmosphere [12]. In general, establishing the relationship between surface moisture and infrared radiation is an underdetermined problem, with many unknown factors that significantly complicate the unambiguous interpretation of the data obtained [13]. During the construction of dams, the problem of flooding forces to search for the possibility of building a mathematical model for determining the potential destructibility of hydraulic engineering objects [14].

\section{MATERIALS AND METHODS}

The object of the study is the dam at the Big Almaty Lake, which is located in the mountains, about $15 \mathrm{~km}$ south of Almaty. The Big Almaty Lake is a reservoir, the purpose of which is power engineering, water supply and artificial irrigation of lands. The dam has the shape of a ledge with a height of about $6 \mathrm{~m}$, the length of the object under study is $250 \mathrm{~m}$, and the width is $6 \mathrm{~m}$. Since the dam provides drinking water to some settlements of Almaty, access to the territory of the lake is closed and strictly guarded. Experimental studies were carried out using the Loza B ground-penetrating radar in order to identify the internal structure of the dam, leakage (humidification) zones, and also to prevent future emergencies. The research was carried out jointly with employees of the Department for Emergency Situations of the city of Almaty of the Ministry of Internal Affairs of the Republic of Kazakhstan.
The result of the GPR survey is a set of single tracks (signals) recorded by the receiving antenna at each position of the radar. As a rule, this set of tracks is displayed by the variable density method in the form of an image - a radiogram. The subject of the research is methods of interpretation of radar images obtained by sounding and diagnostics using a GPR. Experimental studies were carried out using the Georadar Loza B device along the top and along the foot of the dam with $300 \mathrm{~cm}$ antennas $(300 \mathrm{MHz}), 50 \mathrm{~cm}$ with a profile spacing:

- Profile surveys were carried out along the foot of the dam with separate profiles in two directions with a spacing of $50 \mathrm{~cm}$. In the opposite direction, they were carried out by connecting the antennas in one row, tying one antenna to the end of the other antenna (Fig. 1). The survey was conducted in an eastward direction, in Figure 1 it is marked with a red arrow, and in the opposite direction, which is marked with a blue arrow. The profile is about 250 meters long.

- In the upper part of the dam, profile surveys were carried out with separate profiles in two directions with a spacing of $50 \mathrm{~cm}$ (Fig. 2). Profile length is about 250 meters.

GPR surveys in the upper part of the dam were carried out in an eastward direction, which is marked in Fig. 2 with a black arrow, and the westward direction is marked with a purple arrow.

In Figure 2, a square of concrete with an iron pipe in the centre is indicated by a green arrow, a yellow arrow indicates the distance between two iron pillars, and an orange arrow indicates a concrete slab lying on its side. In

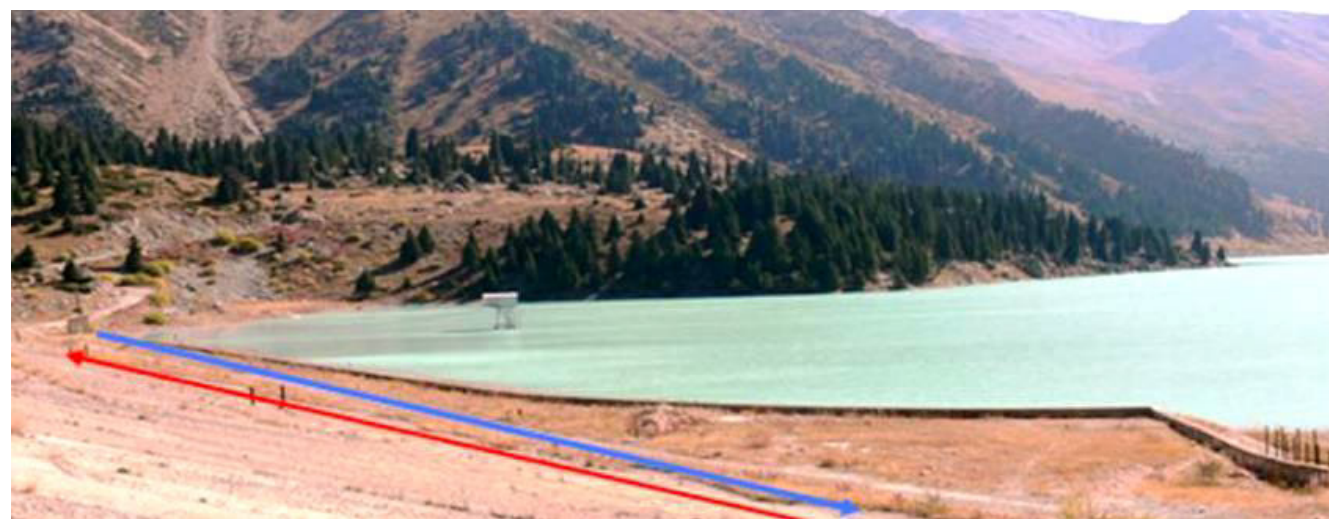

Figure 1: Reservoir dam

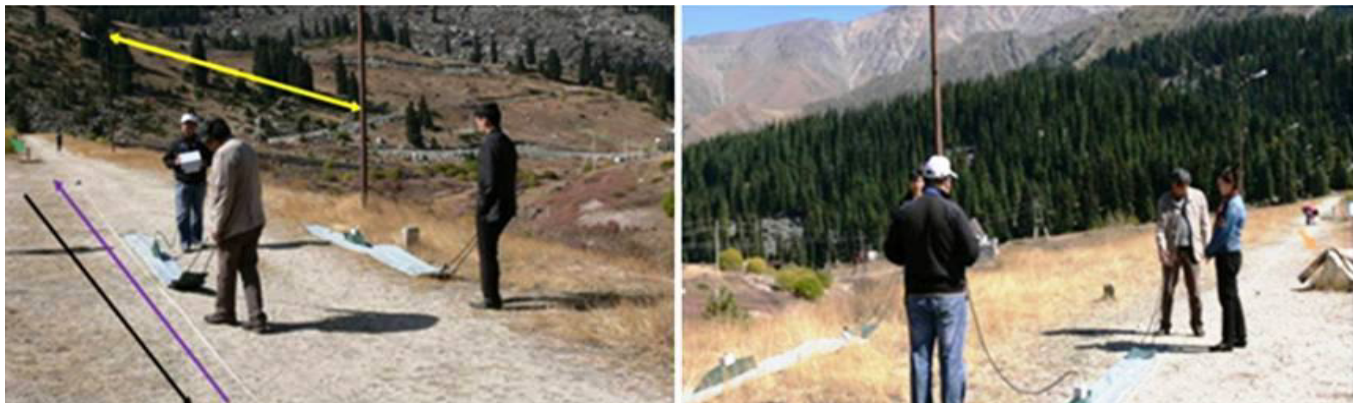

Figure 2: Dam top 


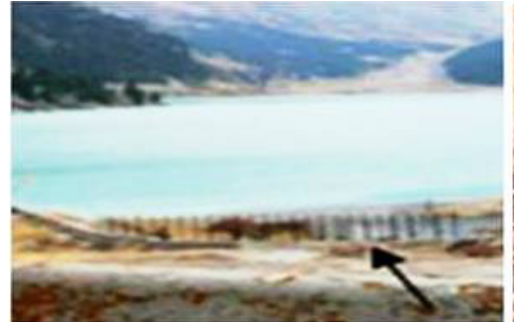

(a)

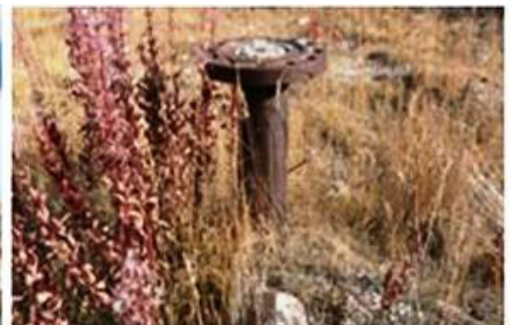

(b)

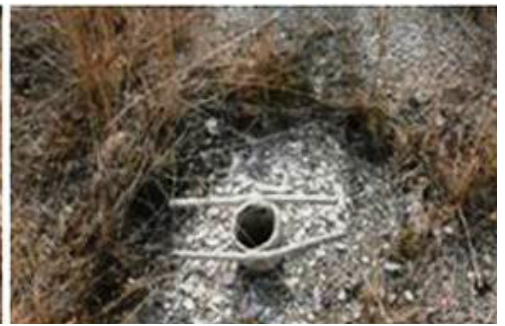

(c)

Figure 3: Surface and subsurface objects: a) reinforcing rods protruding from the ground; b) a pipe with a round end; c) H-shaped pipe

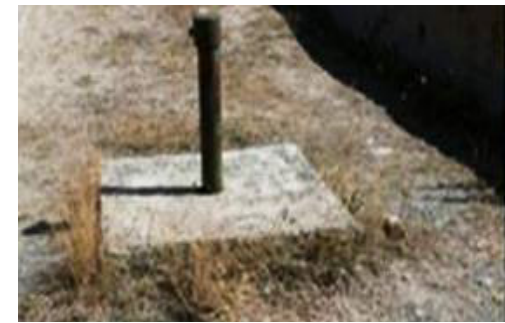

(a)

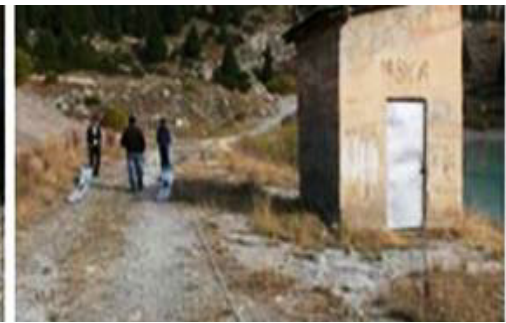

(b)

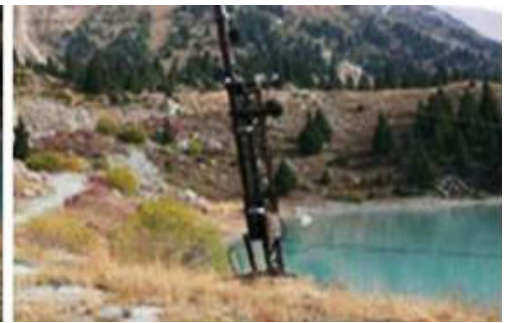

(c)

Figure 4: Surface objects: a) concrete square with an iron pipe in the centre; b) a cabin; c) a pole with a power line

addition, there are many surface and subsurface objects, many of which are shown in Figures 3-4.

\section{RESULTS AND DISCUSSION}

The information obtained by the side-looking radar of the $8 \mathrm{~mm}$ range of the complex is presented in the form of bitmap image files of the reflected radio signal from the earth surface. But in contrast to bitmap images of optical systems, which have minimal geometric distortions in cartographic bitmaps [15], it has much more prominent geometric distortions. It is caused by physical processes that accompany the acquisition of information and the specifics of the operation of the equipment for receiving and recording information from the side-looking radar. This greatly complicates the interpretation of the information received [16].

There are a lot of factors causing the appearance of geometric distortions of bitmap information. These include short-term deviations of the trajectory of the movement of airflows from the rectilinear direction of movement (yaw, pitch), short-term changes in the flight speed, short-term and static changes in the orientation of the aircraft relative to the direction of movement (angle of compensation of wind drift), the specifics of the operation of transceiving and recording equipment of a specific implementation of a side-looking radar [17]. Each of these factors has a different contribution to the result of distortions, but the main ones are distortions due to the specifics of the operation of the equipment and static changes in orientation relative to the direction of movement (hereinafter referred to as drift). It is these factors that must be eliminated at the stage of preliminary processing to prepare materials for further thematic processing of bitmap information [18].
The distortions caused by the specifics of the operation of the equipment include the addition of telemetric and navigational digital information to the structure of the bitmap image, mirror scanning when registering information, distortion of the proportionality of the bitmap due to differences in the horisontal and vertical interpixel distance, uneven interpixel distances in the direction perpendicular to the direction of movement, the presence in the bitmap image of a section of reflected signals up to the first reflected from the earth's surface [19].

The specificity of the operation of the receiving and recording equipment of a specific implementation of an IR radiometer differs significantly from a side-looking radar. The initial information of the IR radiometer does not have a clear bitmap structure as in the case of RPON. This is due to the fact that the recording equipment operates independently of the receiving equipment and the quantisation of analogue information (digitisation) occurs completely asynchronously with the frequency of its own clock generator, which has nothing to do with the receiving equipment. In turn, the receiving equipment has its own frequencies of the sweep formation, which are synchronised by the mechanical sensors of the antenna system of the radiometer, have a free-play, detonation effect and dependence on mechanical vibration of the equipment [20]. The line length of the original image is not an integer multiple. In addition, if we examine in detail the position of the beginning and end of the lines, it can be clearly seen that the instability of the beginning of the line and the asynchronous position of the end of the lines relative to their beginning, that is, the local instability of the length of the lines with sufficiently stable average values of these parameters. The task of preliminary processing of bitmap radar information is to correct the 
existing geometric distortions and generate bitmap information images suitable for integration into geographic information systems and further thematic processing using interactive and automatic methods.

At the first stage of correction, it is necessary to separate the area of the radar image from the area of the service digital information of navigation and telemetry and to make a mirror overturn of the image line elements. To perform this procedure, the input bitmap is represented in the form of a two-dimensional array of data values $A_{(x, y)}$ (input) (where $x$ - address of an element inside the input information line, $\mathrm{y}$ - address of the line) of size NxxNy (where $\mathrm{Nx}$ - the number of elements in the line of the input bitmap, and $\mathrm{Ny}$ - the number of lines of the input bitmap). From this array, it is necessary to form an original array of values $A_{(k, y)}$ (output) (where $k$ - address of an element within the original information line, $\mathrm{y}$ - address of the line) of size Nkx$\mathrm{Ny}$ (where $\mathrm{Nk}$ - the number of elements in the line of the original bitmap image, and $\mathrm{Ny}$ - the number of lines of the original bitmap image). The first 256 bytes of each line of the input image contain service navigation and telemetry information, and, therefore, the number of elements in the line of the original bitmap image must be $\mathrm{Nk}=\mathrm{Nx}-256$. Considering the need to reverse the address of the image line elements, the element $A_{(k=0, y)}$ (output) must correspond to the element $A_{(x=(N x-1), y)}$ (input), that is, the addresses of the radar image elements in the input and output arrays are related by the (Eq. 1).

$x=N x-1-k$

As a result, the procedure for selecting the area of the radar image and flipping the image line is reduced to performing the operation (Eq. 2):

$A_{(k, y)}^{(\text {output })}=A_{((N x-1-k), y)}^{(\text {input })} \quad\left\{\begin{array}{l}k=0 \ldots(N k-1) \\ y=0 \ldots(N y-1)\end{array}\right.$

At the second stage of geometric correction, it is necessary to linearise the space-time scan of the image inside the information line. To perform this procedure, the incoming (after performing the selection of the radar image) bitmap information image is represented in the form of a two-dimensional array of values $A_{(k, y)}$ (input) (where $k$ - address of an element inside the input information line, $y$ - address of the line) of size $N k \times N y$ (where $N k$ - the number of elements in the line of the input bitmap, and $\mathrm{Ny}$ - the number lines of the input bitmap). From this array it is necessary to form an original array of values $A_{(j, y)}{ }_{\text {(output) }}$ (where $j$ - address of the element inside the original information line, $y$ - address of the line) of size $\mathrm{Nj} \times \mathrm{Ny}$ (where $\mathrm{Nj}$ - the number of elements in the line of the original bitmap, and $\mathrm{Ny}$ - the number of lines of the original bitmap).

A feature of the implementation of the onboard digital processing system of the radar is the formation of a controlled delay in the registration of digitised information $t_{0}$, in order to exclude an uninformative original section before the first reflected signal from the registration process. This delay is set by the operator before starting work and can be set both to the minimum position, at which the value for the original element of the line is $t_{0} \leq(2 \mathrm{H} / \mathrm{c})$, and in the position at which the value for the original element of the line is $t_{0}>(2 \mathrm{H} / \mathrm{c})$. In hardware, this is expressed in the shift of the value of the time reference index by the value (Eq. 3):

$t_{0}=t_{0} / d t=\left|t_{0} f_{A Z P}\right|$

Then the address of the elements inside the string $k$ of the input array $A_{(k, y)}$ (input) can be linked to the index of the time reference i (Eq. 4):

$i=\left|t_{0} f_{\text {AZP }}\right|+k$

The interpixel distance $\mathrm{db}$ in the linearised information line depends on the number of elements in the line $\mathrm{m}$ and the size of the information line on the Earth's surface $B$ (Eq. 5), and using trigonometric functions, it can be written as (Eqs. 6-7):

\section{$d b=B / m$}

$B=b_{i=n}=H \operatorname{tg}\left(\operatorname{arcxos}\left(2 H /\left(c t i=t_{n}\right)\right)\right) t_{0} \leq(2 H / c)$

$B=b_{i=n}-b_{i_{0}}=H\left(\operatorname{tg}\left(\arccos \left(2 H /\left(c t_{i=n}\right)\right)\right)-\operatorname{tg}\left(\arccos \left(2 H /\left(c t_{0}\right)\right)\right)\right) t_{0}>(2 H / c)$

Given that $i$ is the index of the time reference, $t_{i}$ can be expressed as (Eq. 8):

$t_{i}=i d t=i / f_{A Z P}$

Since $A_{i=n}$ is the last digitised value, the value of $n$ is determined from the values of $\mathrm{Nk}$ and $\mathrm{t}_{0}$ taking into account (Eqs. 3-4):

$n=i_{0}+(N k-1)=\left|t_{0} f_{A Z P}\right|+(N k-1)$

Then, taking into account (Eqs. 8-9), equations $(6,7)$ take the form (Eqs. 10-11):

$B=H \operatorname{tg}\left(\arccos \left(2 H /\left(c\left(\left|t_{0} f_{A Z P}\right|+(N k-1)\right) / f_{A Z P}\right)\right)\right) t_{0} \leq(2 H / c)$

$B=N\left(\begin{array}{l}\operatorname{tg}\left(\arccos \left(2 H /\left(c\left(\left|t_{0} f_{A Z P}\right|+(N k-1)\right) / f_{A Z P}\right)\right)\right)- \\ -\operatorname{tg}\left(\arccos \left(2 H /\left(c t_{0} f_{A Z P} / f_{A Z P}\right)\right)\right)\end{array}\right) t_{0}>(2 H / c)$

Eqs. 10-11 contain all defined variables and are used to calculate the value of $B$. The value of the index $m$ (Eqs. 7-8) determines the number of elements in the line of the original bitmap (Eq. 12):

$m=N j-1$

Since the interpixel distance in the line of the original bitmap $\mathrm{db}$ is a constant value, which is calculated by (5), the distance along the surface to each distance element can be calculated as (Eqs. 13-14):

$b_{j}=j d b \quad t_{0} \leq(2 H / c)$

$b_{j}=b_{0}+j d b=\left(H \operatorname{tg}\left(\arccos \left(2 H /\left(c t_{0} f_{A Z P} / f_{A Z P}\right)\right)\right)\right)+j d b t_{0}>(2 H / c)$

Each of the elements of the original line $A_{(j, y)}$ (output) is located in space and time between two values (previous and next) 
of the input line $A_{(k, y)}{ }^{\text {(input) }}$ which allows to define its value as the sum of these two values with significance inversely proportional to the distances in space and time from the position of this element to the positions of the previous and subsequent range elements in the input string.

The location of the elements of the original line in space is determined by the (Eqs. 13-14), and the location in time can be determined based on the trigonometric ratio (Eq. 15):

$t_{j}=(2 H / c) /\left(\cos \left(\operatorname{arctg}\left(b_{j} / H\right)\right)\right)$

Using (Eq. 8), it is possible to go to the value of the element index in the original stream of digitisation (Eq. 16):

$t_{j}=f_{A Z P}(2 H / c) /\left(\cos \left(\operatorname{arctg}\left(b_{j} / H\right)\right)\right)$

Using (Eq. 4), it is possible to go to the value of the element index in a given line of the array $A_{(k, y)}$ (input) $($ Eq. 17):

$t_{j}=f_{A Z P}(2 H / c) /\left(\cos \left(\operatorname{arctg}\left(b_{j} / H\right)\right)\right)-\left|t_{0} f_{A Z P}\right|$

In general, the value $K_{j}$ is a real value with a fractional part, which determines the location of this value between two integer values of the indices $k$ and $k+1$ of the el-

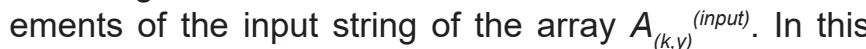
case, the significance is determined as (Eq. 18):

$\left\{\begin{array}{c}a_{k+1}=\left(k_{j}-k_{j}\right) \\ a_{k}=1-a_{k+1}\end{array}\right.$

Taking into account (Eqs. 5-18), the procedure for linearising the space-time scan of the image inside the information line is reduced to performing the operations (Eq. 19):

$\left\{\begin{array}{l}d b=\left(H \operatorname{tg}\left(\arccos \left(2 H /\left(c\left(t_{0} f_{A Z P}+(N k-1)\right) / f_{A Z P}\right)\right)\right)\right) /(N j-1) \\ k_{j}=A_{A Z P}(2 H / c) /\left(\cos \left(\operatorname{arctg}\left(\left(\left(H \operatorname{tg}\left(\arccos \left(\begin{array}{l}2 H / \\ /\left(c t_{0} f_{A Z P} / f_{A Z P}\right)\end{array}\right)\right)\right)+j d b\right) / H\right)\right)\right)-t_{0} f_{A Z P} \\ \left.A_{(j, y)}^{(\text {output })}=\left(1\left(k_{j}-k_{j}\right)\right)\right) A_{\left(k_{j}, y\right)}^{(\text {input })}+\left(k_{j}-k_{j}\right) A_{\left(\left(k_{j}+1\right), y\right)}^{(\text {input })}\end{array}\right.$

for (Eqs. 20-22):

$$
\begin{aligned}
& \left\{\begin{array}{l}
t_{0} \leq(2 H / c) \\
j=0 \ldots(N j-1) \\
y=0 \ldots(N y-1)
\end{array}\right. \\
& \left\{\begin{array}{l}
d b=\left(H \left(\operatorname{tg}\left(\arccos \left(2 H /\left(c\left(t_{0} f_{A Z P}+(N k-1)\right) / f_{A Z P}\right)\right)\right)-\right.\right. \\
\left.\left.-\operatorname{tg}\left(\arccos \left(2 H /\left(c t_{0} f_{A Z P} / f_{A Z P}\right)\right)\right)\right)\right) /(H j-1) \\
b_{j}=\left(H \operatorname{tg}\left(\arccos \left(2 H /\left(c t_{0} f_{A Z P} / f_{A Z P}\right)\right)\right)\right)+j d b \\
k_{j}=f_{A Z P}(2 H / c) /\left(\cos \left(\operatorname{arctg}\left(b_{j} / H\right)\right)\right)-t_{0} f_{A Z P} \\
A_{(j, y)}^{(\text {output })}=\left(1-\left(k_{j}-k_{j}\right)\right) A_{\left(k_{j}, y\right)}^{(\text {input })}+\left(k_{j}-k_{j}\right) A_{\left(\left(k_{j}+1\right), y\right)}^{(i n p u t)}
\end{array}\right.
\end{aligned}
$$

$$
\left\{\begin{array}{c}
t_{0}>(2 H / c) \\
j=0 \ldots(N j-1) \\
y=0 \ldots(N y-1)
\end{array}\right.
$$

At the third stage of geometric correction, it is necessary to bring the line spacing values to the value corrected at the previous stage of the interpixel distance inside the information line, taking into account the spatio-temporal geometry of the propagation of the emitted and reflected signal in the azimuthal plane during earth sensing.

To perform this procedure, represent the incoming (after linearisation) bitmap information image in the form of a two-dimensional array of values $A_{(j, y) \text { (input) }}$ (where $j$ - address of an element inside the input information line, $y-$ address of the line) of size $\mathrm{Nj} \times \mathrm{Ny}$ (where $\mathrm{Nj}$ - the number of elements in the line of the input bitmap, and $N y$ - the number of lines of the input bitmap). From this array, it is necessary to form an original array of data values $A_{(j, s)}$ (output) (where $j$-address of an element inside the original information line, $s$ - address of the line) of size $N j \times N s$ (where $\mathrm{Nj}$ - the number of elements in the line of the original bitmap, and $N s$ - the number of lines of the original bitmap). In the general case, in the presence of a wear angle $\varphi$ the final (after drift correction) value of the interpixel distance will be less than the value $d b$ calculated at the previous stage and will be (Eq. 23):

$d b s=d b \cos (\varphi)$

Taking into account the relationship between the line frequency $f_{s t r}$, the line spacing $d t_{s}$, line spacing on the earth's surface $d L v$ and speed $V$, the vertical length of the input bitmap is (Eq. 24):

$$
L v=d L v(N y-1)=\left(V / f_{s t r}\right)(N y-1)
$$

The number of lines of the original bitmap in this case (Eq. 25):

$$
N s=(L v / d b s)+1=\left(\left(V / f_{s t r}\right)(N y-1) / d b s\right)+1
$$

The line index of an input image element determines its location in $/ v_{y}$ space (Eq. 26):

$v_{y}=d L v y$

The line index of an input image element determines its location in $I_{s}$ space (Eq. 27 ):

$I v_{s}=d b s s$

For each value of the index $s$ calculate the location of the element in space, and then, through the position in space, calculate the values of the index $y$ (Eq. 28):

$y_{s}=/ v_{s} / d L v=(d b s s) /\left(V / f_{s t r}\right)$

In general, the value $y_{s}$ is a real value with a fractional part, which determines the location of this value between two integer values of the indices $y$ and $y+1$ of the elements of the input row of the array $A_{(i, y)}$ (input). In this case, the significance is determined as (Eq. 29): 


$$
\left\{\begin{array}{c}
a_{y+1}=\left(y_{s}-y_{s}\right) \\
a_{y}=1-a_{y+1}
\end{array}\right.
$$

Given (Eqs. 23-29), the procedure for aligning the line and interpixel spacing is reduced to performing the following operations (Eq. 30):

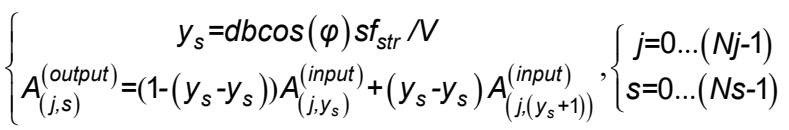

At the fourth stage of geometric correction, it is necessary to carry out a wear correction. To perform this procedure, represent the input (after linearisation and correction of the line spacing) bitmap information image in the form of a two-dimensional array of data values $A_{(j, s)}^{\text {(input) }}$ (where $j$ - address of an element inside the input information line, $s-$ the address of the line) of size $N j \times N s$ (where $N j$ - the number of elements in the line of the input bitmap, and $\mathrm{Ns}$ - the number of lines of the input bitmap). From this array, it is necessary to form an original array of values $A_{(j, p)}$ (output) (where $j$ - address of an element inside the original information line, $p$ - address of the line) of size $N j \times N p$ (where $\mathrm{Nj}$ - the number of elements in the line of the original bitmap, and $N p$ - the number of lines of the original bitmap).

To correct this curvature, it is necessary to perform a vertical shift of the output bitmap columns by an amount proportional to the number of the element in the row so that for the last, at $\varphi \geq 0$ (the first at $\varphi<0$ ) column of the bitmap, the shift would be (Eq. 31):

\section{$L s=B \sin (\varphi)$}

In this case, the vertical size of the output file increases and the number of lines $N p$ will be (Eq. 32):

$N p=N s+(B \sin (\varphi)) / d b s$

The shift amount for each element of the input image column will be (Eqs. 33-34):

$$
\begin{aligned}
& d s_{j}=j B \sin (\varphi) /(N j-1) \quad \varphi \geq 0 \\
& d s_{j}=(N j-1-j) B \sin (\varphi) /(N j-1) \quad \varphi<0
\end{aligned}
$$

Then the address of the line $p$ of the original array $A_{(j p)}$ (output) can be associated with the address of the line $s$ of the input array $A_{(j, s)}^{\text {(input) }}$ (Eqs. 35-36):

$$
\begin{aligned}
& s_{p}=p-d s_{j}=p-j B \sin (\varphi) /(N j-1) \quad \varphi \geq 0 \\
& s_{p}=p-d s_{j}=p-(N j-1-j) B \sin (\varphi) /(N j-1) \quad \varphi<0
\end{aligned}
$$

In general, the value $s_{p}$ is a real value with a fractional part, which determines the location of this value between two integer values of the indices $s$ and $s=1$ of the input array string $A_{(j, s)}{ }^{\text {(input) }}$. The value of an element of the original array $A_{(j . p)}$ (joutput) is determined as the sum of the values of the

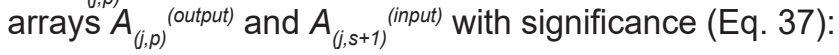

$\left\{\begin{array}{c}a_{s+1}=\left(s_{p}-s_{p}\right) \\ a_{s}=1-a_{s+1}\end{array}\right.$
Considering (Eqs. 31-37), the wear adjustment procedure is reduced to performing the following operations (Eq. 38):

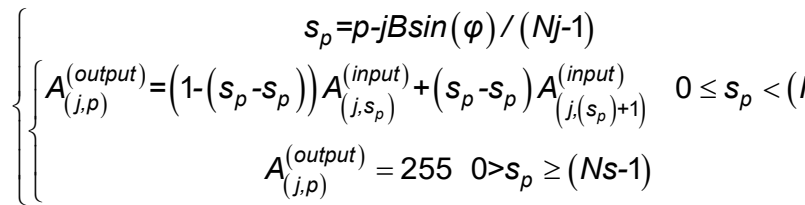

For (Eqs. 39-41):

$$
\begin{aligned}
& \varphi \geq 0 \\
& j=0 . . .(N j-1) \\
& p=0 . . .(N P-1) \\
& \left\{\begin{array}{c}
s_{p}=p-(N j-1-j) B \sin (\varphi) /(N j-1) \\
\left\{\begin{array}{c}
A_{(j, p)}^{(\text {output })}=\left(1-\left(s_{p}-s_{p}\right)\right) A_{\left(j, s_{p}\right)}^{(\text {input })}+\left(s_{p}-s_{p}\right) A_{\left(j,\left(s_{p}+1\right)\right)}^{(\text {input })} 0 \leq s_{p}<(N s- \\
A_{(j, p)}^{(\text {output })}=255 \quad 0>s_{p} \geq(N s-1)
\end{array}\right.
\end{array}\right.
\end{aligned}
$$

$$
\left\{\begin{array}{c}
\varphi<0 \\
j=0 \ldots(N j-1) \\
p=0 \ldots(N p-1)
\end{array}\right.
$$

Based on the above algorithms, a software was developed that performs the correction of geometric distortions. The programme allows to load the input data file, which immediately after loading is displayed in the left graphics window, set the value of the input file data line size (Input total string size, pix), the value of the image line size in the input file data line (Input image string size, pix), the value of the offset of the original image element in the data line of the input file (Input image string offset, pix), the value of the quantisation frequency of the analogue-to-digital converter (Fatc, $\mathrm{Hz}$ ), the value of the frequency of the lines of the recording system (Fstr, Hz), the value of the original registration delay $\left(t_{0}, s\right)$, flight altitude $(\mathrm{H}, \mathrm{m})$, flight speed $(\mathrm{V}, \mathrm{km} / \mathrm{h})$, drift angle (Drift, degree), Output image string size (pix).

In the process of preparing data for correction, the programme calculates and outputs to the information windows the value of the swath over the surface in the angular plane $(B, m)$, the value of the interpixel distance for a given size of the output image line $(\mathrm{db}, \mathrm{m})$, the value of the interpixel distance for a given line size the original image taking into account the drift angle ( $\mathrm{dbs}, \mathrm{m})$, the value of the number of lines of the input image (Input image size, string) and the value of the number of lines of the original image taking into account the drift angle (Output image size, string). After visual control of the calculated parameters, the programme performs a geometric correction ("CORR") and display the result in the right graphic window.

The space-time geometry of the scan process of the Earth's surface with an IR radiometer shows that the points of time $t_{i}$ correspond to the points of quantisation (digitisation) of the analogue signal from the output of the $\mathrm{IR}$ radiometer, starting from the start of scanner opera- 
tion. The receiving equipment of the IR radiometer scans the earth's surface with a narrowly directed diagram according to the equilibrium time law, that is, the change in the viewing angle of the antenna system is directly proportional to time and with a constant sampling frequency of the output analogue signal in direct proportion to the time of the sampling (digitisation) instant. At the same time, the distance from the start to the sighting point is defined as (Eq. 42):

$$
b=H \operatorname{tg}(\alpha)
$$

where $b$ - the distance along the surface of the earth from the beginning to the irradiation point, $\mathrm{H}$ - position of the antenna above the earth's surface, $\alpha$ - angle between the beginning and the direction to the point of irradiation. Then for each moment of quantisation it is possible to determine the distance over the surface from the beginning to the sighting point (Eq. 43):

$$
b_{i}=H \operatorname{tg}\left(\alpha_{i}\right)
$$

Considering that the angle of sight $\alpha$ changes linearly in time, starting from value $-\theta / 2$ to value $+\theta / 2$, with a step $d \theta$ the value of which is determined as (Eq. 44):

$$
d \theta=\theta / n
$$

where $\theta$ - angle of the scanning strip, $n$ - value of the number of digitised scans reduced by one, and the value of the angle $\alpha_{i}$ is determined as (Eq. 45):

$\alpha_{i}=i d \theta-\theta / 2=(i \theta / n)-\theta / 2, \quad i=0 \ldots n$

Then (Eq. 43) can be written as (Eq. 46):

$b_{i}=H \operatorname{tg}((i \theta / n)-\theta / 2), \quad i=0 \ldots n$

It can be seen from (Eq. 46) that with a linear change in the values of $t_{i}$ the value of $b_{i}$ change nonlinearly, and, accordingly, the values of interpixel distances change nonlinearly in the direction perpendicular to the direction of motion, which are defined as $b_{i+1}-b$, which is the reason for the appearance of geometric distortions of bitmap information images expressed in unevenness of interpixel distances in the direction perpendicular to the direction of travel. Distortions of the bitmap image of the IR radiometer in the azimuthal plane due to the angles of wear and the dependence of the line spacing on the flight speed have the same nature and laws as for bitmap images RPON.

At the first stage of correction, it is necessary to normalise the bitmap structure of the information image. To do this, it is necessary to search for the beginning of the information line along the trailing edge of the blanking pulse, which has a maximum signal amplitude and a fixed duration from a short sample pulse of zero amplitude to information samples. Then it is necessary to search for the end of the information line by the difference from the low level of the zero-signal section at the end of the information line to the high level of the blanking pulse. Next, it is necessary to normalise the length of the information line and perform interline filtering followed by compression (the number of output lines is reduced by 2 times) to minimise distortions associated with changing the location of information when normalising the size of the line and proceed to analyse and normalise the next information line. After that, it is necessary to perform the inversion of the signal amplitude and write the normalised bitmap structure of the information image to the original file.

To perform the procedures described above, a software has been developed that runs in console mode. For the programme to work correctly, an information file must be completed. The programme allows processing not only the entire input file, but also its fragment, and also performs amplitude correction, converting 16-bit data (12 effective bits for an IR radiometer) to an 8-bit representation, suitable for further processing by applications that operate 8-bit data.

At the second stage of geometric distortion correction, it is necessary to linearise the space-time scan of the image inside the information line. To perform this procedure, represent the incoming (after the procedure for normalising the bitmap) bitmap image in the form of a two-dimensional array of values $y-A_{(i, y)}$ (input) (where $i$ - address of an element inside the input information line, $y$ - address of the line) of size $\mathrm{Ni} \times \mathrm{Ny}$ (where $\mathrm{Ni}$ - the number of elements in the line of the input bitmap, and $\mathrm{Ny}-$ number of lines of input bitmap). From this array it is necessary to form an original array of values $A_{(j, y)}{ }^{\text {(output) (where }}$ $j$-address of the element inside the original information line, $y$-address of the line) of size $\mathrm{Nj} \times \mathrm{Ny}$ (where $\mathrm{Nj}$ - the number of elements in the line of the original bitmap, and $\mathrm{Ny}$ - the number of lines of the original bitmap).

Another feature of the specific implementation of the receiving equipment of the radiometer is that the number of input image elements corresponding to the angle of the scanning strip $\theta=84^{\circ}$ is less than the length of the information line $\mathrm{Ni}$ (the strip at the end of the image lines does not carry useful information, but is an element of the information line) and has the value $\mathrm{Nn}$. Therefore, the equation for calculating activities on the surface (Eq. 46) for elements of the input image takes the form (Eq. 47):

$b_{i}=H \operatorname{tg}((i \theta /(N n-1))-\theta / 2) \quad i=0 \ldots(N n-1)$

The scan line for surface $B$ is defined as (Eq. 48):

\section{$B=2 H \operatorname{tg}(0 / 2)$}

In the general case, the values $B_{i}$, calculated by (Eq. 46) have negative values for $i<(N n / 2)$, and the initial absolute value is equal to half the scan line over the surface (Eq. 49):

$$
b_{i=0}=-(B / 2)=-(H \operatorname{tg}(\theta / 2))
$$

By shifting the origin of the distance along the surface to a point $\mathrm{t}_{\mathrm{i}=0}\left(\mathrm{~b}_{\mathrm{i}=0}\right)$ obtain (Eq. 50):

$$
b_{i}=H(\operatorname{tg}((i \theta /(N n-1))-\theta / 2)+\operatorname{tg}(\theta / 2)) \quad i=0 \ldots(N n-1)
$$

Each of the elements of the original line $A_{(j, y)}$ (output) is located in space and time between two values (previous and next) of the input line $A_{(i, y)}{ }^{\text {(input) }}$ which allows to determine 
its value as the sum of these two values with significance inversely proportional to the distances in space and time from the position of this element of the previous and subsequent range elements in a given row. The location of the elements of the original line in space is determined by (Eq. 51):

$b_{j}=j d b \quad j=0 \ldots(N j-1)$

where (Eq. 52):

$d b=B /(N j-1)=2 H \operatorname{tg}(\theta / 2) /(N j-1)$

The location of the elements of the original line in time can be determined by the equation, inverse to (Eq. 50):

$i_{j}=((\theta / 2)+\operatorname{arctg}((j d b / H)-\operatorname{tg}(\theta / 2)))(N n-1) / \theta \quad j=0 \ldots(N j-1)$

In general, a value $i_{i}$ is a real value with a fractional part, which determines the position of this value between two integer values of the indices $i$ and $i+1$ elements of the input string of the array $A_{(i, y)}$ (input). In this case, the significance is determined as (Eq. 54):

$\left\{\begin{array}{c}a_{i+1}=\left(i_{j}-i_{j}\right) \\ a_{i}=1-a_{i+1}\end{array}\right.$

Taking into account (Eqs. 51-54), the procedure for linearising the space-time scanning of the image inside the information line is reduced to performing the operations (Eq. 55).

$$
\left\{\begin{array}{c}
d b=2 H \operatorname{tg}(\theta / 2) /(N j-1) \\
i_{j}=((\theta / 2)+\operatorname{arctg}((j d b / H)-\operatorname{tg}(\theta / 2))) \\
A_{(j, y)}^{\text {(output) }}=\left(1-\left(i_{j}-i_{j}\right)\right) A_{\left(i_{j}, y\right)}^{(\text {input })}+\left(i_{j}-i_{j}\right) A_{\left(\left(i_{j}+1\right), y\right)}^{(\text {input })}
\end{array} \quad(N n-1) / \theta,\left\{\begin{array}{c}
j=0 \ldots(N j-1) \\
y=0 \ldots(N y-1)
\end{array}\right.\right.
$$

At the third stage of correcting distortions, it is necessary to bring the value of the line spacing to the value corrected at the previous stage of the interpixel spacing within the information line. And at the fourth stage of correcting distortions, it is necessary to correct the wear. The algorithms of these stages fully correspond to the algorithms of the similar stages of the RPON bitmap correction. Files containing corrected bitmap information images are transferred for further thematic processing.

\section{CONCLUSIONS}

In order to prevent emergency situations, experimental studies are being carried out on geophysical mapping of the Big Almaty Lake dam. The internal structure of the dam, the zone of leakage have been determined. The experimental results were processed in the embedded software of the GPR. Various methods were used to interpret the radiogram, such as filtering data from noise, hodograph diagram, and depth determination method.

In general, based on the appearance of the profile (direction of layers, hyper-period from objects), it can be argued that the dam is dry at a depth of 12-14 meters. If the dam leaks, it takes place deep underground due to high pressure. Further experimental research is recom- mended, the profile needs to be made longer, and it is necessary to go outside the dam to investigate the geology around the edges.

\section{ACKNOWLEDGMENTS}

The authors express their deep gratitude to Prof., Dr. A. Berdyshev and PhD N. Akhtaeva for advice on experimental research.

The work supported by the Ministry of Education and Science of the Republic of Kazakhstan, under the Grant No. 132, 12 March 2018 (IRN: AP05133922).

\section{REFERENCES}

1. Dicaire, I., Jukna, V., Praz, C., Milián, C., Summerer, L., Couairon, A. (2016). Spaceborne laser filamentation for atmospheric remote sensing. Laser and Photonics Reviews, vol. 10, no. 3, 481-493. DOI: 10.1002/lpor.201500283

2. Fryirs, K.A., Brierley, G.J., Hancock, F., Cohen, T.J., Brooks, A.P., Reinfelds, I., Cook, N., Raine, A. (2018). Tracking geomorphic recovery in process-based river management. Land Degradation and Development, vol. 29, no. 9, 3221-3244. DOI: 10.1002/ldr.2984

3. Lunga, D., Ersoy, O. (2011). Kent mixture model for classification of remote sensing data on spherical manifolds. 2011 IEEE Applied Imagery Pattern Recognition Workshop (AIPR). DOI: 10.1109/ AIPR.2011.6176337

4. He, X., Stamnes, K., Bai, Y., Li, W., Wang, D. (2018). Effects of Earth curvature on atmospheric correction for ocean color remote sensing. Remote Sensing of Environment, vol. 209, 118-133. DOI: 10.1016/j. rse.2018.02.042

5. Meng, X.-Y., Che, L., Liu, Z.-H., Che, N., Ji, X.-N. (2017). Towards a partial differential equation remote sensing image method based on adaptive degradation diffusion parameter. Multimedia Tools and Applications, vol. 76, no.17, 17651-17667. DOI: 10.1007/ s11042-015-2881-1

6. Radecki-Pawlik, A., Kidová, A., Lehotsky, M., Rusnák, M., Manson, R., Radecki-Pawlik, B. (2019). Gravel and boulders mining from mountain stream beds. E3S Web of Conferences, 106, article number 01005. DOI: 10.1051/e3sconf/201910601005

7. An, X., Sun, Q., Yang, Y., Gong, H. (2013). A method for extracting area water body from remote sensing images using active contour model and vector data. Geomatics and Information Science of Wuhan University, vol. 38, no. 10, 1152-1157.

8. Yu, L., Porwal, A., Holden, E.-J., Dentith, M.C. (2012). Towards automatic lithological classification from remote sensing data using support vector machines. Computers and Geosciences, vol. 45, 229239. DOI: 10.1016/j.cageo.2011.11.019 
9. Ahmad, N.A., Goh, P.S., Yogarathinam, L.T., Zulhairun, A.K., Ismail, A.F. (2020). Current advances in membrane technologies for produced water desalination. Desalination, 493, article number 114643. DOI: 10.1016/j.desal.2020.114643

10. Hui, L., Lei, P., Lei, Z., Xin, H., Jinying, L., Kexin, L., Changfeng, J., Wei, L. (2014). A cooperation earth observation model of SAR satellite and optical remote sensing satellite. International Geoscience and Remote Sensing Symposium (IGARSS). DOI: 10.1109/IGARSS.2014.6946490

11. Chang, S.-T., Ho, C.-E., Yang, M.-Y., Hung, H.-C., Huang, T.-M., Chen, C.-R. (2012). Design and analysis of the multispectral remote sensing imager. Proceedings of SPIE - The International Society for Optical Engineering. https://www.spiedigitallibrary.org/conference-proceedings-of-spie/8528/1/ Design-and-analysis-of-the-multispectral-remote-sensing-imager/10.1117/12.977394.short?S$\mathrm{SO}=1 \&$ tab $=$ ArticleLink

12. Wang, X., Sun, L., Wan, Y., Wang, S., Tao, J. (2016). Remote sensing image inpainting based on non-local sample filling and adaptive curvature driven diffusions model. Pattern Recognition and Artificial Intelligence, vol. 29, no. 8, 735-743. DOI: 10.16451/j.cnki. issn1003-6059.201608008

13. Pradhan, B., Lee, S., Mansor, S., Buchroithner, M., Jamaluddin, N., Khujaimah, Z. (2008). Utilization of optical remote sensing data and geographic information system tools for regional landslide hazard analysis by using binomial logistic regression model. Journal of Applied Remote Sensing, vol. 2, no. 1, article number 023542. DOI: 10.1117/1.3026536

14. Luo, W., Wang, W., Lang, F., Gui, G. (2012). Ship detection of remote sensing image on FRHT and multi-points curvature based polygon approximation. Research Journal of Applied Sciences, Engineering and Technology, vol. 4, no. 15, 2590-2599.
15. Pradhan, B., Youssef, A.M. (2010). Manifestation of remote sensing data and GIS on landslide hazard analysis using spatial-based statistical models. Arabian Journal of Geosciences, vol. 3, no. 3, 319-326. DOI: 10.1007/s12517-009-0089-2

16. Yang, K., Jia, Y., Shen, C. (2020). Haze and cloud removal from remote sensing image using HTM algorithm based on curvature filtering. Journal of Image and Graphics, vol. 25, no. 4, 791-800. DOI: 10.11834/jig. 190309

17. Li, H., Zhong, C., Huang, X., Li, D. (2012). Automatic overpass detection with LiDAR and remote sensing image. Acta Geodaetica et Cartographica Sinica, vol. 41, no. 3, 428-433.

18. Silva, J.D., Fernandes, V., Limont, M., Dziedzic, M., Andreoli, C.V., Rauen, W.B. (2020). Water sustainability assessment from the perspective of sustainable development capitals: Conceptual model and index based on literature review. Journal of Environmental Management, 254, article number 109750.

19. Salama, M.S., Verhoef, W. (2015). Two-stream remote sensing model for water quality mapping: 2SeaColor. Remote Sensing of Environment, vol. 157, 111-122. DOI: 10.1016/j.rse.2014.07.022

20. Shi, X., Li, X., Zheng, C. (2010). New encryption approach based on ECC for remote sensing images. Geomatics and Information Science of Wuhan University, vol. 35, no.11, 1309-1313. 